\title{
Kernos
}

Revue internationale et pluridisciplinaire de religion grecque antique

3 | 1990

Varia

\section{La théorie de l'inspiration prophétique dans les dialogues pythiques de Plutarque}

\section{Yvonne Vernière}

\section{OpenEdition \\ Journals}

Édition électronique

URL : http://journals.openedition.org/kernos/1007

DOI : 10.4000/kernos.1007

ISSN : 2034-7871

Éditeur

Centre international d'étude de la religion grecque antique

Édition imprimée

Date de publication : 1 janvier 1990

ISSN : 0776-3824

Référence électronique

Yvonne Vernière, "La théorie de l'inspiration prophétique dans les dialogues pythiques de Plutarque », Kernos [En ligne], 3 | 1990, mis en ligne le 19 avril 2011, consulté le 30 avril 2019. URL : http:// journals.openedition.org/kernos/1007; DOI : 10.4000/kernos.1007 


\section{LA THÉORIE DE L'INSPIRATION PROPHÉTIQUE DANS LES DIALOGUES PYTHIQUES DE PLUTARQUE}

Depuis 90 environ jusqu'à sa mort en 125, Plutarque fut prêtre de Delphes. C'est dire qu'il était familier de l'adyton, qu'il assista à d'innombrables consultations de Pythies successives, dont il était le supérieur hiérarchique, et que, par conséquent, ses réflexions sur l'oracle présentent pour nous un intérêt exceptionnel. Or deux faits caractéristiques de son époque l'ont frappé. D'une part beaucoup d'oracles étaient alors en pleine décadence ou même réduits au silence. D'autre part, si la Pythie continuait son office, elle ne s'exprimait plus en vers mais en prose. Ces deux remarques servent de point de départ à deux dialogues célèbres qui se déroulent à l'intérieur du sanctuaire, l'un Sur la disparition des oracles (De defectu oraculorum), l'autre Pourquoi la Pythie ne rend plus ses oracles en vers abrégé dans le titre latin De Pythiae oraculis. Mais Plutarque, ou plutôt les devisants qu'il met en scène, profitent de l'occasion pour aborder le problème d'ensemble de la mantique inspirée, celui-là même qui nous intéresse ici.

Attention! Il ne s'agit pas d'une mise en question de la véracité de l'oracle ni de l'authenticité de la transe pythique. Les soupçons qui pèseront de Lucien de Samosate jusqu'à Fontenelle sur une éventuelle fraude sacerdotale sont tout à fait étrangers à Plutarque : «N'écoutons pas, dit-il ceux qui prétendent qu'il y a des oracles dépourvus de toute inspiration divine». Et cette foi inconditionnelle est plus vive encore lorsqu'il s'agit de la divination enthousiaste, bien supérieure aux divinations inductives (cléromancie, hiéroscopie, clédonomancie, ornithomancie, etc.) et surtout lorsqu'il s'agit de l'oracle de Delphes, le sien.

Mais Plutarque ne se contente pas d'affirmer l'authenticité et le caractère sacré de l'inspiration prophétique. Il voudrait en trouver la cause, en démonter le mécanisme. Or le problème est de taille : comment se fait le passage d'une réalité spirituelle à une expression verbale concrète ? Quels intermédiaires permettent ce passage du spirituel au matériel ?

Il faut reconnaître que le genre platonicien du dialogue convient bien mieux à une enquête de ce type qu'un exposé dogmatique. Chaque devisant a sa personnalité et éclaire une facette du problème. Ainsi 
Lamprias, frère de Plutarque, principal narrateur du De defectu oraculorum, est connu par ailleurs comme un philosophe dans la ligne d'Aristote. Dans son long exposé à la première personne, qui remplit 13 chapitres du dialogue, on reconnaît des traits péripatéticiens et stoïciens. Il insistera sur les facteurs physiologiques et les influences physiques qui peuvent expliquer la transe prophétique. En revanche, le Lacédémonien Cléombrote, grand voyageur à tendance mystique, cédera volontiers aux prestiges de l'imagination. D'où son goût pour les explications démonologiques. Quant à Ammonios, qui fut le maitre de Plutarque, ainsi que le sage Théon du De Pythiae oraculis, ils semblent bien être les porte-parole de l'auteur et ont en général le dernier mot.

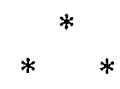

Le De defectu oraculorum est une conversation de philosophes, typique d'une pensée qui se cherche. Deux principales doctrines vont y être examinées tour à tour, dont aucune ne s'avèrera pleinement satisfaisante, mais qui n'en présentent pas moins un vif intérêt : l'une est naturaliste, l'autre démonologique.

L'exaltation du prophète et du devin a très tôt intrigué les philosophes grecs par son caractère irrationnel. Dans un remarquable article de l'Antiquité classique de 1934, A. Delatte montrait comment Héraclite, Empédocle, Démocrite avaient tenté d'en élucider les causes. Jeanne Croissant, à son tour, dans son livre Aristote et les mystères, a étudié avec beaucoup de pénétration le point de vue d'Aristote. Toutes ces doctrines étaient familières à Plutarque, ainsi que les idées stoïciennes sur la divination. Aussi met-il dans la bouche de Lamprias toutes sortes d'arguments en faveur d'une théorie naturaliste de l'inspiration prophétique.

Le postulat, que l'on trouve déjà chez Aristote, avec quelques nuances, c'est que l'inspiration n'est pas réservée à des créatures exceptionnelles; c'est une faculté innée chez tous les humains, mais elle est d'ordinaire obscurcie par l'union de l'âme et du corps. Elle ne se manifeste que dans les songes ou à l'heure de la mort, c'est-à-dire dans des moments priviligiés où, dégagée à la fois des sens et de la raison, l'âme s'ouvre quelque peu à des représentations irrationnelles, et d'ailleurs indistinctes, de l'avenir. Ici intervient, à titre de preuve, une comparaison curieuse entre la mémoire et la divination (432a) qui semble une intuition quasi bergsonienne de la théorie du subconscient : il faut bien que la mémoire retienne de façon mystérieuse les choses qui 
ne sont plus, puisque celles-ci affleurent en certains cas. De même, pourquoi la faculté prophétique ne garderait-elle pas à l'état latent les choses qui ne sont pas encore? On comprend dès lors que la Pythie ne soit pas choisie pour des dons médiumniques particuliers. C'est une simple fille de Delphes, peu instruite, élevée chez des paysans, et dont on n'exige qu'une grande pureté de mœurs. Elle ne porte pas partout avec elle un don personnel, comme jadis Cassandre. La transe ne la saisit qu'à des moments déterminés et dans l'espace strictement limité de l'adyton. Il faut donc supposer l'intervention nécessaire d'agents appropriés, qui vont éveiller cette faculté engourdie, et permettre le passage de la puissance à l'acte, pour parler comme Aristote.

Il y a d'abord une mise en condition au moyen de cérémonies propitiatoires préliminaires. La Pythie secoue le laurier sacré; elle en mâche les feuilles vertes, en respire les feuilles brûlées avec de l'orge. Elle fait ses ablutions à la fontaine Castalie et boit à la source Cassotis. Bien sûr, on s'est demandé si le laurier avait des effets physiologiques, toxiques ou hallucinogènes; et Dodds nous dit plaisamment que le Professeur Oesterreich s'est contraint à mâcher une grande quantité de feuilles de laurier et qu'il ne s'est pas senti plus inspiré qu'à l'ordinaire. De même l'eau de Cassotis n'a aucune vertu particulière. Mais ces rites devaient provoquer une puissante auto-suggestion. De même la cérémonie consistant à arroser une chèvre d'eau froide et à n'autoriser la consultation que si elle frémissait de tous ses membres n'a apparemment aucun rapport avec la prophétie. Et pourtant Plutarque nous rapporte (438b) un épisode curieux dont il fut témoin. La chèvre n'ayant pas réagi, les prêtres forcent néanmoins la Pythie à monter sur le trépied pour satisfaire des consultants de marque. Celle-ci paraît bouleversée, pousse un cri terrible, tombe évanouie et meurt peu après. On peut penser que la pauvre femme est terrassée par l'angoisse d'avoir violé le tabou. Les spirites actuels considèrent d'ailleurs qu'il y a danger à forcer un médium qui agit à contrecœur. On a connu, paraît-il, des cas mortels. Tout cela prouve l'importance, non pas matérielle, mais psychologique, de ces rites propitiatoires. En ce sens on peut dire, comme Marie Delcourt, que "la source et le laurier sont des conditions du miracle".

Puis la Pythie monte sur le trépied. La tradition veut que celui-ci soit placé sur une crevasse du sol, un "chasma" ou un «stomion" d'où s'échappent des vapeurs, et que ce soient ces vapeurs qui provoquent la transe. Strabon appelle ce souffle pneuma enthousiastikon (IX, 3, 5), Cicéron afflatus terrae, le pseudo-Longin (XIII, 2) atmon entheon, etc. Tous semblent l'accepter comme une réalité matérielle. D'ailleurs une 
légende, contée tout au long par Diodore (XVI, 25) et dont Plutarque se fait ici l'écho, en explique l'origine. En des temps très anciens, le berger Corétas gardait ses chèvres près d'une crevasse, à l'endroit où se trouva plus tard l'adyton. Chaque fois qu'une chèvre approchait de la crevasse, elle se mettait à bondir et à bêler de façon particulière. Le berger s'approche à son tour; il est saisi d'enthousiasme et se met à prophétiser. Après que plusieurs personnes sont tombées dans le gouffre en état de délire, on décide de fermer le site et de réserver le "pneuma» à une seule prophétesse. Plutarque, à vrai dire, fait des réserves : «Ce récit n'est-il pas une fable ou une invention gratuite ? Pour ma part je le crois» dit-il (435d). Bien plus; dans un article remarquable du Bulletin de Correspondance hellénique de 1942-1943 sur La nature du pneuma delphique, E. Will montre que nulle part Plutarque n'affirme positivement l'existence d'exhalaisons matérielles à cet endroit. Sans doute, en $437 \mathrm{c}$, il fait état d'un parfum suave qui "au témoignage de certains étrangers et de tous les serviteurs du sanctuaire" envahit parfois l'adyton. Mais puisque ces anathumioseis - ces "odeurs de sainteté» si l'on veut - se produisent "non pas fréquemment ni régulièrement mais à intervalles fortuits», on peut penser que, même si elles existent, elles ne peuvent être déterminantes dans des transes prophétiques fréquentes et régulières. D'ailleurs les archéologues modernes ont été bien déçus, comme on sait, de ne trouver sur place ni crevasses, ni exhalaisons!

Cependant Lamprias attribue un rôle déterminant à ce qu'il appelle lui aussi le pneuma. Mais, pour le définir, c'est aux doctrines des philosophes qu'il fait appel, et non à la tradition delphique. Les souvenirs de Démocrite, d'Héraclite (cité en 432f), d'Aristote (cité en 434b), de la cosmologie vitaliste des Stoïciens, bien que tacite et diffuse, ne sont guère contestables ici. Ces doctrines admettent toutes qu'il n'y a pas de véritable différence de nature entre la matière et l'esprit, si bien que le «souffle ou effluve divinatoire" (mantikon pneuma kai reuma) peut pénétrer dans le corps et se mêler à l'âme avec laquelle il a "parenté et affinité». Il s'ensuit un «mélange» (krasis) entre ces deux matières subtiles. Le pneuma «se propage soit directement par l'air soit indirectement par l'intermédiaire d'un liquide». Par un effet de dilatation, il uouvre certains pores qui donnent entrée aux images de l'avenir» (on retrouve ici Démocrite). Le processus peut s'entendre aussi comme une dessication qui échauffe et purifie (ceci se rapproche d'Héraclite) ou encore comme une réfrigération et une condensation qui trempe l'âme comme le fer (ce serait le choix des Stoïciens). Mais de toutes façons l'enthousiasme s'explique, comme chez Aristote, par des 
facteurs physiques. Aussi Lamprias envisage-t-il réciproquement que des facteurs physiques puissent, à leur tour, entraver ou détruire l'action du pneuma : "Les pluies diluviennes l'étouffent, la foudre le dissout, les séismes le refoulent». Ce qui peut expliquer par des raisons météorologiques la décadence de certains oracles.

Mais le pneuma peut-il souffler partout ? En réalité l'originalité du site delphique demeure : «Les exhalaisons d'ici sont les seules à disposer les âmes à l'enthousiasme». Nous assistons donc, malgré sa réactualisation par les doctrines des philosophes, à la persistance d'un vieux fonds religieux tellurique. En outre, comme le suggère BouchéLeclerq, il faut peut-être voir là un effort de propagande sacerdotale qui lie volontairement l'inspiration prophétique spirituelle à un espace sacré strictement localisé.

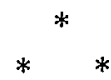

$\grave{A}$ cette théorie naturaliste et pseudo-scientifique s'oppose l'explication démonologique de Cléombrote. Au lieu de supposer une faculté dormante exaltée par des facteurs extérieurs physiques, celui-ci revient en fait à la vieille doctrine selon laquelle toute altération psychique est attribuable à la possession du médium par un être surnaturel venu d'ailleurs. Croyance populaire fort ancienne, relayée notamment par Hésiode et par les Pythagoriciens. Mais Platon est passé par là. Les démons ne sont plus désormais des êtres mystérieux et redoutables qui errent à travers le monde «vêtus de brume». Ce sont des esprits purs, intermédiaires entre dieux et hommes, qui servent aux dieux de «secrétaires» et d'«interprètes» dans toutes les cérémonies religieuses. On peut donc supposer que certains d'entre eux sont plus spécialement chargés de la divination et des oracles. Ainsi, tout en étant les fidèles truchements de la sagesse divine, ils évitent aux dieux une intervention directe indigne de leur majesté. En outre, comme ils sont mortels (on se souvient de la mort du "grand Pan»), leur disparition peut expliquer opportunément la mort de certains oracles. Sans eux, en effet, ces oracles "restent comme des instruments de musique devenus inutiles et muets» (431b). Quant au mécanisme de la possession, il reste assez flou. Il s'expliquerait, selon Cléombrote, par l'identité de nature entre âmes humaines et esprits démoniques : «C'est par la différence existant entre un homme quelconque et un homme qui joue la tragédie que l'on peut se figurer celle qui sépare un pur esprit d'un esprit qui, revêtu d'un corps, se trouve mêlé à la vie présente" (431c). La 
pénétration des uns par les autres n'a donc rien, théoriquement du moins, de surprenant ni d'absurde.

Aucune de ces deux théories ne satisfait Ammonios, porte-parole de Plutarque. En effet, insister ainsi sur le rôle décisif des intermédiaires physiques ou démoniques, n'est-ce pas déplacer la causalité dans une perspective tout empirique et même athée, et réduire à rien ou à presque rien le rôle du dieu dans son oracle ? En vain Lamprias se défend en distinguant les "causes finales", indispensables pour déclencher le processus et l'enchaînement des "causes secondes" qui en découle. Ammonios considère que le débat reste ouvert et propose en conclusion de remettre à plus tard l'étude de ces questions car, dit-il, «elles suscitent beaucoup d'objections et d'hypothèses contradictoires que nous n'avons pas maintenant le loisir de passer toutes en revue».

Le débat n'est repris qu'une vingtaine d'années plus tard dans le $D e$ Pythiae oraculis, qui est probablement la dernière œuvre de Plutarque octogénaire; et il est repris dans une optique différente, non plus philosophique, mais religieuse. L'un des devisants pose bien le problème : "Il convient de chercher la solution des contradictions apparentes et de ne pas abandonner la piété et la foi de nos pères» (403c).

S'exprimant par la bouche de Théon, Plutarque supprime les intermédiaires et ne garde en présence que deux personnages : le dieu et la Pythie. Il s'agit simplement dès lors de préciser leur rôle respectif dans une ambiance tout apollinienne.

Sans pneuma, sans démons, le dieu agit directement sur la prophétesse. Plus précisément, «il donne l'impulsion et le mouvement et provoque les visions de cette femme en produisant dans son âme la lumière qui éclaire pour elle l'avenir. C'est en cela que consiste l'enthousiasme (enthousiasmos, 397c). La Pythie est donc passive. Elle ne sert au dieu que d'instrument (organon). Mais la qualité d'un instrument n'est pas indifférente pour la beauté de la musique. Il doit "se conformer le mieux possible, selon les ressources qui lui sont propres, à l'agent qui l'emploie» (404c). De même la Pythie doit être aussi simple, aussi transparente que possible : issue d'une famille 
honorable, de mœurs pures, mais "pleine d'ignorance et d'inexpérience», elle est ouverte au divin en raison inverse de son intellectualité. "C'est avec une âme encore vierge qu'elle s'approche du dieu» (parthenos tèn psychèn) comme une jeune mariée de son époux. Rien d'oriental dans son allure : «Elle descend dans le lieu prophétique sans s'être parfumée, sans avoir revêtu d'étoffes de pourpre, et elle ne brûle, au lieu de cannelle, de laudanum et d'encens, que du laurier et de la farine d'orge» (397a). Rien de dionysiaque non plus, contrairement à ce :que pensait Erwin Rohde. Ni vin, ni danse échevelée dans les préparatifs de la consultation. Et si, dans le De defectu oraculorum, Lamprias comparait l'enthousiasme à l'ivresse bachique, le pneuma pénétrant par les pores "à la façon des vapeurs du vin», Théon, lui marque nettement la différence (406b) entre l'ivresse des buveurs qui dénature l'individu et l'enthousiasme prophétique qui, comme celui des poètes et des amoureux, l'utilise sans l'altérer. Il s'agit en somme d'une transe irrationnelle, mais nullement frénétique. Et de comparer la Pythie à ces oiseaux, hérons, corbeaux et roitelets, que l'on considérait parfois comme des messagers des dieux.

Cependant Plutarque insiste sur le fait qu'un oracle n'est pas une épiphanie. La Pythie est au dieu ce que la lune est au soleil, un reflet, un miroir. Aussi les conceptions du dieu arrivent-elles «adultérées par le passage à travers un corps mortel et une âme humaine». En conséquence, le dieu n'est pas responsable de la formulation de l'oracle. Non seulement celle-ci varie selon la personnalité des Pythies (certaines d'entre elles, paraît-il, prophétisaient en patois phocidien), mais elle varie aussi selon les données sociologiques. Ainsi l'expression versifiée s'accordait avec une mentalité primitive mais (406d) «il survient dans le cours des choses et les tempéraments des hommes des changements" qui modifient les mentalités. Voilà pourquoi les oracles contemporains de Plutarque sont rendus en prose, sans que leur valeur en soit en rien diminuée.

Faut-il conclure avec Robert Flacelière que Plutarque, à la fin de sa vie, a évolué au point de remplacer la réflexion philosophique par la foi aveugle ? Les choses ne sont pas si simples. N'oublions pas qu'il se réclame de Platon et que, dans le Phèdre (244c), Socrate déclarait : «Les plus grands des biens sont ceux qui nous viennent par l'intermédiaire d'un délire (mania) à condition que celui-ci nous soit accordé par un 
don divin». Et de donner une place de choix, auprès des poètes et des amoureux, aux devins et notamment à la Pythie, cause de tant de bienfaits pour la Grèce dans son délire, alors que «dans son bon sens son action se réduisait à presque rien ou même à rien du tout». On conviendra qu'une telle affirmation, qui trouve son écho dans le Timée (71a), de la supériorité de l'irrationnel comme moyen de connaissance, n'a rien à voir avec une crédulité naïve.

Par ailleurs Plutarque, s'il renonce à disséquer en médecin, comme le faisait Aristote, le mécanisme physiologique de l'enthousiasme, n'en reconnaît pas moins l'efficacité du rituel préparatoire et la présence de facteurs externes qui déterminent, dans une certaine mesure, le psychisme de la Pythie. Elle vit dans un monde à part, contrainte à la continence, presque prisonnière dans la maison du dieu et, pour parler comme Marie Delcourt, "elle parle dans un état second où la met, non les prêtres qui l'auraient hypnotisée, mais le lieu auguste dont elle est une sorte d'abrégé». Les modernes parleraient d'auto-suggestion. Plutarque se contente d'insister sur le caractère foncièrement religieux de cet enthousiasme qu'Alfred Guillaume compare, non sans raison, avec celui des prophètes d'Israël.

Il n'est pas jusqu'à la démonologie qui ne laisse des traces; mais il s'agit moins ici d'une croyance que de poésie. Pierre Boyancé, dans la Revue d'études anciennes de 1938, s'attachait au passage du De Pythiae oraculis où les devisants aperçoivent, près de la fontaine Cassotis, un sanctuaire dédié aux Muses «assistantes et gardiennes de la divination". Il suggère que ces "démones", sans être les ouvrières de l'oracle, assurent en quelque sorte le mélange musical entre le souffle d'Apollon et son instrument humain.

Ainsi Plutarque ne renie rien, ni la réflexion philosophique et psychologique, ni la gracieuse allégorie mythologique; mais il est certain que, dans cet état ultime de sa pensée, la foi dépasse et intègre tout cela dans la pure lumière apollinienne.

Rue de Bretagne, 35

Yvonne VERNIÈRE F - 92600 ASNIÈRES 